\title{
Using historical dip net data to infer absence of flatwoods salamanders in stochastic environments
}

\author{
George C Brooks ${ }^{\text {Corresp., } 1}$, Carola A Haas ${ }^{1}$ \\ ${ }^{1}$ Department of Fish and Wildlife Conservation, Virginia Polytechnic Institute and State University (Virginia Tech), Blacksburg, Virginia, United States \\ Corresponding Author: George C Brooks \\ Email address: boa10gb@vt.edu
}

Local extinction and undetected presence are two very different biological phenomena, but they can be challenging to differentiate. Stochastic environments hamper the development of standardized monitoring schemes for wildlife, and make it more challenging to plan and evaluate the success of conservation efforts. To avoid reintroductions of species at risk that could jeopardize extant populations, managers attempting translocation events require a higher level of confidence that a failure to confirm presence represents a true absence. For many pond breeding amphibians, monitoring of the breeding population occurs indirectly through larval surveys. Larval development and successful recruitment only occurs after a sequence of appropriate environmental conditions, thus it is possible for a breeding population of adults to exist at a site but for detectability of the species to be functionally zero. We investigate how annual variability in detection influences longterm monitoring efforts of Reticulated Flatwoods Salamanders (Ambystoma bishopi) breeding in 29 wetlands in Florida. Using 10 years of historic dip net data, we simulate plausible monitoring scenarios that incorporate environmental stochasticity into estimates of detection probability. We found that annual variation in environmental conditions precluded a high degree of certainty in predicting site status for low-intensity monitoring schemes. Uncertainty was partly alleviated by increasing survey effort, but even at the highest level of sampling intensity assessed (four surveys per year), multiple years of monitoring are required to confidently determine presence/absence at a site. Combined with assessments of habitat quality and landscape connectivity, our results can be used to identify sites suitable for reintroduction efforts. Our methodologies can be generally applied to increase the effectiveness of surveys for diverse organisms for which annual variability in detectability is known. 
1 USING HISTORICAL DIP NET DATA TO INFER

2 ABSENCE OF FLATWOODS SALAMANDERS IN

3 STOCHASTIC ENVIRONMENTS

4

5 George C. Brooks ${ }^{1}$ and Carola A. Haas ${ }^{1}$

6

7

$8 \quad{ }^{1}$ Department of Fish and Wildlife Conservation, Virginia Tech, 310 West Campus Drive,

9 Blacksburg VA 24060

10

11 Corresponding Author:

12 George Brooks ${ }^{1}$

13310 West Campus Drive, Blacksburg VA 24060

14 Email address: boa10gb@vt.edu 


\section{Abstract}

18 Local extinction and undetected presence are two very different biological phenomena, but they

19 can be challenging to differentiate. Stochastic environments hamper the development of

20 standardized monitoring schemes for wildlife, and make it more challenging to plan and evaluate

21 the success of conservation efforts. To avoid reintroductions of species at risk that could

22 jeopardize extant populations, managers attempting translocation events require a higher level of

23 confidence that a failure to confirm presence represents a true absence. For many pond breeding

24 amphibians, monitoring of the breeding population occurs indirectly through larval surveys.

25 Larval development and successful recruitment only occurs after a sequence of appropriate

26 environmental conditions, thus it is possible for a breeding population of adults to exist at a site

27 but for detectability of the species to be functionally zero. We investigate how annual variability

28 in detection influences long-term monitoring efforts of Reticulated Flatwoods Salamanders

29 (Ambystoma bishopi) breeding in 29 wetlands in Florida. Using 10 years of historic dip net data,

30 we simulate plausible monitoring scenarios that incorporate environmental stochasticity into

31 estimates of detection probability. We found that annual variation in environmental conditions

32 precluded a high degree of certainty in predicting site status for low-intensity monitoring

33 schemes. Uncertainty was partly alleviated by increasing survey effort, but even at the highest

34 level of sampling intensity assessed (four surveys per year), multiple years of monitoring are

35 required to confidently determine presence/absence at a site. Combined with assessments of

36 habitat quality and landscape connectivity, our results can be used to identify sites suitable for

37 reintroduction efforts. Our methodologies can be generally applied to increase the effectiveness

38 of surveys for diverse organisms for which annual variability in detectability is known. 


\section{Introduction}

41 Local extinction and undetected presence are two very different phenomena, but they can be

42 challenging to differentiate. There has been much attention devoted to detection probability, and

43 especially to the challenges raised when working with rare and cryptic species in stochastic

44 environments (Lebreton et al., 1992; MacKenzie et al., 2002; Royle, Nichols \& Kéry, 2005;

45 Pacifici, Dorazio \& Conroy, 2012; Kellner and Swihart, 2014; Moore et al., 2014; Specht et al., 46 2017; Folt et al., 2019). The detectability of many species varies with environmental conditions;

47 annual variation in weather can strongly affect the optimal time of year to survey, and the overall

48 likelihood of detecting the target species (Field, Tyre \& Possingham, 2005; Jackson et al., 2006;

49 McConville et al., 2009; Rizzo et al., 2017; Shaffer, Roloff \& Campa 2019). If the factors

50 influencing detectability are not known, one can never be confident that failure to detect

51 constitutes a true absence, and the development of standardized monitoring schemes will be

52 severely hampered (Penteriani et al., 2005; Bried \& Pellet, 2012; Gervasi et al., 2014; Bellier,

53 Kéry \& Schaub, 2016; Crone, 2016).

54 Species with complex life cycles pose an additional challenge because different life-stages may

55 require different sampling methodologies, or may only be available for capture at particular

56 times of the year. Many pond-breeding amphibians are fossorial for most of the year, migrating

57 to and from breeding sites annually in response to seasonal cues. For such species, it is nearly

58 impossible to avoid monitoring techniques that rely on specific environmental conditions to be

59 effective. Factors known to directly influence detectability are often unknown, difficult to

60 assess, or poorly understood. Thus in many instances, monitoring schemes cannot be

61 strategically tailored to maximize success with any degree of precision. 
62 Amphibian monitoring typically occurs at breeding wetlands, either through drift-fences that

63 intercept arriving individuals, dip-net surveys for aquatic larvae, or egg-mass counts (Wells,

64 2007). Owing to the high labor cost of continually running a drift-fence however, monitoring of

65 adult populations is often sporadic or entirely absent. Population assessments based solely on

66 dip-net data are common, yet fraught with uncertainty (Penteriani et al., 2005; Bried \& Pellet,

67 2012; Gervasi et al., 2014; Bellier, Kéry \& Schaub, 2016; Crone, 2016). Because larval

68 development occurs only in years with suitable weather and hydrological conditions, it is

69 possible for a breeding population of adults to occur but for the detection probability of larvae to

70 be zero. Failure to detect may simply represent a bust recruitment year, offering no information

71 as to the health of the population. In some cases it should be easy enough to realize that the

72 species would be undetectable in a given year (extreme drought), but certain patterns of pond

73 filling and drying and refilling, or flooding carrying in fish predators, may also cause complete

74 egg or larval mortality without being obvious (Semlitsch, 1987; Dodd, 1993; Taylor, Scott \&

75 Gibbons, 2006).

76 In some cases, the repercussions of failing to detect an organism are considerably more severe

77 than incorrectly documenting a presence, i.e., false negatives are more problematic than false

78 positives (Hauser \& McCarthy, 2009; Charney, Kubel \& Eiseman, 2015; Veale \& Russello,

79 2016). Conservation reintroductions are a useful example of the risk imbalance between type-1

80 and type-2 errors. Failure to detect a target organism may result in reintroductions that could

81 jeopardize extant populations (Kéry \& Schmidt, 2008). Introducing animals to an extant

82 population can have positive (Hedrick \& Frederickson, 2010) or negative (Laikre et al., 2010)

83 impacts on genetic viability, and as such a clear distinction must be made between establishing

84 new populations and augmenting existing ones. Identifying suitable, unoccupied sites for 
85 establishing new populations can be hampered by environmental stochasticity. Similarly,

86 evaluating the relative success of reintroduction efforts is made challenging by low or uncertain

87 detectability brought on by environmental variation. Thus there is a clear need for approaches

88 that can confidently predict site status and determine the fate of individuals following

89 reintroduction under a range of environmental conditions.

90 We have monitored hydrology and worked with partners to restore suitable vegetation in

91 wetlands across Eglin Air Force Base, in hopes of restoring Reticulated Flatwoods Salamanders

92 (Ambystoma bishopi) to a greater proportion of their former range. Procedures for allowing an

93 existing population to expand into improved habitat, augmenting an existing population, or

94 conducting a re-introduction are very different (and for a protected species would require

95 different levels of approval from regulatory agencies). Assessing whether a translocation action

96 would result in augmentation vs. re-introduction depends on knowing whether there is an extant

97 population or not. Here we investigate how annual variability in detection influences long-term

98 monitoring efforts. Using historic dipnet data we simulate a range of scenarios that incorporate

99 environmental stochasticity into estimates of detection probability.and evaluate the efficacy of

100 different monitoring schemes. In the face of environmental uncertainty, identifying potential

101 reintroduction sites requires high survey effort over a period of years to confidently declare

102 absence. Our procedure can be adapted for any species when there are data available for

103 detection probability over a series of years that encompass a meaningful range of environmental 104 conditions. 
107 Study site and species. - Our study was conducted on Eglin Air Force Base (Eglin), Okaloosa

108 and Santa Rosa counties, Florida, USA from 2009 to 2017. Eglin is a large military installation

109 (188,459 ha) primarily consisting of actively managed longleaf pine-dominated sandhills

110 (approximately 145,000 ha) interspersed with treeless open test ranges, pine plantations, and

111 mesic flatwoods. The study focused on 29 ephemeral wetlands that have been monitored

112 intensively for Reticulated Flatwoods Salamander larvae since 2009. Reticulated Flatwoods

113 Salamanders are mole salamanders in the family Ambystomatidae and are federally listed as

114 endangered, with restoration efforts underway and reintroduction efforts currently being planned.

115 Individuals migrate in the fall to dry basins, and deposit eggs terrestrially (Palis 1997). Embryos

116 develop so that if winter rains inundate the ponds after several weeks or months, the eggs hatch

117 into aquatic larvae. Wetlands were surveyed for salamander larvae using standard dipnet

118 sampling techniques (Bishop et al., 2006; Heyer et al., 1994; Wilkinson, 2015) between 2009

119 and 2018. Surveyed wetlands range in size from 0.1 ha to 20.9 ha and consist of shallow 120 depressions which fill in the autumn and typically remain inundated throughout the spring

121 (Chandler et al., 2016). To account for imperfect detection, at least three surveys were conducted

122 annually, spaced one per calendar month. Surveys were conducted during the months of January

123 until May, and survey effort was standardized to $30 \mathrm{~min}$ for each occasion (see Brooks et al.,

1242019 for full description of field methods).

125

126 Statistical approach.-Using posterior distributions of year-specific detection probabilities

127 derived from a recent occupancy study (Brooks et al., 2019), we investigated the impact of

128 environmental stochasticity on long-term monitoring of Reticulated Flatwoods Salamanders.

129 Over the study period, detection probabilities conditional on a site being occupied ranged from 
1300.02 to 0.88 for a single survey. We adopted a bootstrapping approach, whereby ten years were

131 randomly drawn from the dataset with replacement to construct a decade-long series of potential

132 detection probabilities. We iterated this procedure 1000 times to generate a sample of

133 hypothetical time-series, reflecting our uncertainty in detection given annual environmental

134 fluctuations. A decade was chosen partly to reflect the study duration from which the original

135 data were drawn, and also to approximate the longevity of the species (Brooks 2020), i.e. if our

136 aim is to determine whether Reticulated Flatwoods Salamanders have been extirpated from a

137 site, we would need to continue monitoring until we were confident that no breeding adults

138 remained on the landscape.

139 We first calculated the detection probabilities and associated uncertainty with increasing survey

140 effort (up to 10 surveys) conducted within a single year. We combined the estimates from all

141 years for which detection was available, corresponding to a situation in which the relationship

142 between environmental conditions and detectability are unknown. We then estimated the

143 likelihood of detecting larvae given a site is occupied under four levels of survey effort.

144 Scenarios ranged from one to four surveys per year and extrapolated out to 10 years of

145 continuous monitoring for each of the 1000 hypothetical detection histories. Cumulative

146 detection probabilities for multiple surveys within years were calculated by taking the exponent

147 of the probability of failing to detect an animal, $(1-p)^{n}$, where $\mathrm{p}$ is the detection probability for

148 a given year and $\mathrm{n}$ is the number of surveys. Cumulative detection probabilities across years are

149 simply one minus the product of each year-specific estimate. This approach requires that a site's

150 occupancy status does not change over the course of monitoring, but given the high site fidelity

151 of this species (Brooks et al. 2019), we consider there to be a negligible probability of emigration

152 from the focal site over that time (i.e. no turnover). From the 1000 scenarios we derived $90 \%$ 
153 credible intervals for detection probabilities to quantify the uncertainty in site status under

154 different levels of sampling intensity and duration. All analyses were conducted in R version

1554.10 (R Core Team 2021). All of the data and code used to perform the analysis and generate the

156 figures are provided as supplementary material.

157

158 Results

159 We found that in the face of environmental uncertainty, increasing the number of intensive, 30-

160 minute surveys in a single year could never achieve detection probabilities above 95\% with

161 confidence (Fig. 1). Unsurprisingly, given such tremendous annual variability in detection and

162 no knowledge of the environmental conditions in a particular year, an assessment of

163 presence/absence cannot be made without multiple years of surveying. Although median

164 detection probability reached 99\% after six surveys, the lower bound for detection probability

165 remained below 90\% even after 10 surveys (Fig. 1), because during drought years the ability to

166 detect larvae can become next to impossible. In contrast the upper confidence limit for detection

167 approaches unity after only three surveys, reflecting years where conditions for detecting larvae

168 are optimal.

169 We further found that even when multiple years of surveys are conducted, a high degree of

170 survey effort within years is necessary to achieve high detection rates (Fig. 2). If sites are

171 sampled only once per year, surveys must be carried out for five years on average to obtain a

172 detection probability above $95 \%$, and seven years to obtain a detection probability above $99 \%$

173 (Fig. 2). Again, given uncertainty in the relationship between environmental conditions and

174 detectability, there are wide confidence bands on these estimates, whereby $99 \%$ probabilities

175 could be achieved in three years or could take a decade, depending on the specific years in which 
176 sampling takes place. If sites are surveyed twice per year detection probabilities above $95 \%$ and

$17799 \%$ can be achieved sooner, at three and five years respectively, and the associated uncertainty

178 in when thresholds are reached is reduced (Fig. 2). Three surveys per year achieved a detection

179 probability above $95 \%$ after only three years of monitoring and $99 \%$ after four years (Fig. 2). If

180 four surveys are conducted each year, 95\% and 99\% thresholds are reached after two and three

181 years respectively, and uncertainty in detection is completely ameliorated following four years of 182 monitoring (Fig. 2).

\section{Discussion}

185 Environmental stochasticity strongly impacts long-term monitoring efforts for Reticulated

186 Flatwoods Salamanders. Even after a decade of sampling, the occupancy status of Reticulated

187 Flatwoods Salamanders cannot be confidently determined if only one survey is conducted each

188 year (Fig. 1). Increasing the number of surveys within a year greatly reduces the uncertainty in

189 cumulative detection, but even under the highest sampling intensity, at least 5 years are required

190 before sites can confidently be declared unoccupied (Fig. 2).

191 Amphibian populations are frequently monitored through dipnet surveys that target larvae

192 following breeding. For Reticulated Flatwoods Salamanders, researchers have surveyed using

193 dipnets two or three times per year (Palis, 1997; Bishop et al., 2006), and we followed protocols

194 described by Gorman, Bishop \& Haas (2009). Standardized monitoring schemes are attractive

195 because they are easy to employ and produce data in a structure desirable for statistical analysis.

196 Such boilerplate schemes however, fail to account for year-to-year fluctuations in survey

197 conditions which prevents a high degree of certainty in predicting site status for low-intensity

198 monitoring schemes. To improve confidence in our own data, we have added a fourth sampling 
199 200

201

202 203

204 205

206 207 208 209 210

211

212

213

214

215

216

217

218

219

220

221

event per season to our protocol, if no flatwoods salamanders were detected after the first three, and have shifted from attempting to sample 25 to $50 \%$ of ponds each year to attempting to sample all ponds annually. This will give us the best chance of having sufficient data over a 10year period to have confidence in our estimates of occupancy, given there will likely be a few years in each decade when drought makes sampling impossible and likely one or two years when financial constraints allow us to only sample $50-75 \%$ of ponds. Because reticulated flatwoods salamanders have a maximum adult lifespan of around 10-12 years, to ensure that the local breeding population has been extirpated, it is necessary to confirming lack of breeding under suitable environmental conditions over at least a 10-year period. In fact, on our study site, we have observed periods of 8 or 9 years of sampling with no detections and then detected larvae again, in ponds that are far (1000 m or more) from other occupied ponds.

The complex life-cycle of Reticulated Flatwoods Salamanders severely inhibits a reliable assessment of population viability from aquatic surveys alone. Zero detection can occur if 1) the ponds are already filled with water when adults arrive, resulting in eggs being laid outside the pond basin and therefore never being inundated, 2) the pond never fills or fills too late for hatching to occur, 3) the pond dries before larvae can be detected, 4) ponds are overrun with predators. Only when complex facets of a species' life history and ecology are well understood can a flexible monitoring scheme be employed that incorporates the likelihood of encountering an individual in the timing and/or intensity of surveys. Anecdotally, in our own study we have observed a 9-year gap between detections, so could have considered certain populations to have been extirpated if we were not aware of these issues. We had two ponds where flatwoods salamander larvae were detected in 2010, but no detections were made for the next 9 years despite annual sampling. Severe summer drying followed by abundant winter rainfall in 2019- 
2222020 created ideal conditions for larval development and we detected larvae at these two ponds 223 again in the spring of 2020.

224 Until recently, employing a dynamic sampling approach would make subsequent analysis and 225 detecting long-term trends more challenging, but modern statistical techniques can readily 226 accommodate such imbalanced data. Charney, Kubel \& Eiseman (2015) describe a method of 227 adaptive sampling that focuses available resources to sample ambystomatid larvae only in years 228 when they are most likely to be detectable, based on conditions in bellwether ponds. Because 229 regional surveys showed high correlation between reproduction within bellwether ponds and 230 other sites, differences in larval abundance were used to determine whether a year was likely to 231 be appropriate for wide scale sampling (N Charney, pers. comm.). However, if breeding 232 attempts are not highly synchronized across the landscape, if bellwether ponds are unavailable, 233 or if climatic conditions that create "good" breeding years are unknown, the approach can be 234 challenging to implement (Pacifici, Dorazio \& Conroy, 2012; Shaffer, Roloff \& Campa 2019). 235 Although confidence that a species is truly absent if it's not detected can be improved if survey 236 effort is increased, the likelihood will also strongly depend on the prior probability of presence 237 (Wintle et al., 2012). This prior could depend on the time since the species was last observed, 238 perceived site suitability, presence of other species at this site or the focal species at neighboring 239 sites on the landscape. Any reintroduction decisions should take into account habitat 240 connectivity, potential future metapopulation dynamics, and long distance dispersal mechanisms.

241 The marked uncertainty in environmental fluctuations and their impact on detection rates

242 necessitate intensive survey effort over many years before a site can be considered for 243 translocated animals. 
244 For other aquatic organisms for which detection is challenging, environmental DNA (eDNA) has

245 been a powerful tool. The annual fluctuations that make detection through dipnetting

246 challenging however, will likely influence eDNA results in the same way. If the ponds are dry,

247 no method that depends on the presence of aquatic larvae will be able to detect them. When

248 conditions are suitable, even using modified techniques of taking frequent local samples and

249 processing larger volumes of water, our colleagues found that eDNA was comparable to our

250 dipnetting techniques, with both detecting salamanders in most ponds, but each failing to detect

251 salamanders in about the same number of occupied sites (Goldberg et al. 2018).

252 Under the ESA, reintroductions are being considered in areas where wild populations have been

253 extirpated to aid recovery of Reticulated Flatwoods Salamanders. In order to identify potential

254 reintroduction sites, intensive, long-term sampling is necessary to provide a high level of

255 confidence that lack of detections reflected true absence. Once new populations have been

256 established, multiple survey methods should be implemented to evaluate the success of

257 reintroduction efforts (He \& Gaston, 2000; Manel, Williams \& Ormerod, 2001; Royle \&

258 Nichols, 2003; Nielson et al., 2005; Royle, Nichols \& Kéry, 2005; Cosentino, 2014). Owing to

259 density dependence in the aquatic stage, larval abundance can seldom be used to infer population

260 dynamics (Shea, Wolf \& Mangel, 2006; Scherer \& Tracy, 2011). Whilst confirming the return

261 of mature individuals to a breeding site, the often weak relationship between larval presence and

262 population processes prevents a robust assessment of long-term viability from dip-net data alone

263 (Strayer, 1999; Gaston et al., 2000; Zhou \& Griffiths, 2007; Conlisk et al., 2009; Korfel et al.,

264 2010; Bried \& Pellet, 2012).

265 This paper does not consider cost as a limitation (Field, Tyre \& Possingham, 2005; Moore et al., 266 2014). It is important to note however, that the cost of larval sampling is orders of magnitude 
267 less than the cost of monitoring adults through drift-fence sampling, because the latter requires 268 installation of infrastructure, nightly monitoring at least every 24 hours for an extended period of

269 time (or repeated removal and replacement of traps over weeks or months of sampling), and a 270 large enough crew to monitor all sites at the same time. The strategies of stopping surveys after 271 detection (Regan et al., 2006; Rout, Heinze \& McCarthy, 2010; Guillera-Arroita, Hauser \& 272 McCarthy, 2014) or determining occupancy through eDNA may reduce monitoring costs, but do 273 not readily translate to conservation applications when other management objectives (such as 274 assessing growth rates, obtaining genetic samples from a population, etc.) necessitate captures of 275 multiple individuals.

276

\section{Conclusions}

278 In conclusion, annual variability in environmental conditions can severely affect the results of 279 standardized long-term amphibian monitoring efforts. Ideally, multiple sampling methods would 280 be employed to discern population trends or distinguish between local extirpation and undetected 281 presence, but this is often cost-prohibitive. Given the longevity of ambystomatid salamanders, 282 coupled with their cryptic lifestyles, a high survey effort across multiple years is required to infer 283 absence, particularly when sites are being evaluated for future reintroductions. The

284 methodologies outlined here will aid other researchers in tailoring monitoring schemes to 285 overcome the confounding effects of environmental stochasticity.

\section{Acknowledgements}

288 We would like to thank the team of people who have made this research possible. Special 289 mention should be given to John Palis, David Bishop, and Thomas Gorman for establishing the 
290 dipnetting protocol and database, as well as to Lourdes Oztolaza, Kelly Jones, Brandon Rincon, 291 Jay Parker, Steve Goodman, Vivian Porter, and the myriad technicians involved in data 292 collection. 


\section{References}

294 Bellier E, Kéry M, Schaub M. 2016. Simulation-based assessment of dynamic N-mixture models

295 in the presence of density dependence and environmental stochasticity. Methods in Ecology and 296 Evolution 7:1029-1040.

297 Bishop DC, Palis JG, Enge KM, Printiss DJ, Stevenson DJ. 2006. Capture rate, body size, and 298 survey recommendations for larval Ambystoma cingulatum (Flatwoods Salamanders).

299 Southeastern Naturalist 5:9-17.

300 Bried JT, Pellet J. 2012. Optimal design of butterfly occupancy surveys and testing if occupancy 301 converts to abundance for sparse populations. Journal of Insect Conservation 16:489-499.

302 Brooks GC, Smith JA, Frimpong EA, Gorman TA, Chandler HC, Haas CA. 2019. Indirect 303 connectivity estimates of amphibian breeding wetlands from spatially explicit occupancy 304 models. Aquatic Conservation: Marine and Freshwater Ecosystems 29:1815-1825.

305 Chandler HC, Rypel AL, Jiao Y, Haas CA, Gorman TA. 2016. Hindcasting historical breeding 306 conditions for an endangered salamander in ephemeral wetlands of the southeastern USA:

307 Implications of climate change. PLoS One 11:e0150169.

308 Charney ND, Kubel JE, Eiseman CS. 2015. Temporally adaptive sampling: A case study in rare 309 species survey design with marbled salamanders (Ambystoma opacum). PLoS One 10:e0120714.

310 Conlisk E, Conlisk J, Enquist B, Thompson J, Harte J. 2009. Improved abundance prediction

311 from presence-absence data. Global Ecology and Biogeography 18:1-10.

312 Cosentino BJ. 2014. Monitoring plan to detect trends in occupancy of Illinois chorus frogs

313 (Pseudacris streckeri illinoensis). Illinois Department of Natural Resources, Springfield, Illinois, 314 USA. 
315 Crone EE. 2016. Contrasting effects of spatial heterogeneity and environmental stochasticity on 316 population dynamics of a perennial wildflower. Journal of Ecology 104:281-291.

317 Cyr H. 1997. Does inter-annual variability in population density increase with time? Oikos $318 \quad 79: 549-558$

319 Dodd Jr CK. 1993. Cost of living in an unpredictable environment: the ecology of striped newts 320 Notophthalmus perstriatus during a prolonged drought. Copeia 605-614.

321 Field SA, Tyre AJ, Possingham HP. 2005. Optimizing allocation of monitoring effort under

322 economic and observational constraints. Journal of Wildlife Management 69:473-482.

323 Folt B., McGowan CP, Steen DA, Piccolomini S, Hoffman M, Godwin JC, Guyer C. 2019.

324 Modeling strategies and evaluating success during repatriations of elusive and endangered 325 species. Animal Conservation doi:10.1111/acv.12537

326 Gaston KJ, Blackburn TM, Greenwood JJ, Gregory RD, Quinn RM, Lawton JH. 2000.

327 Abundance-occupancy relationships. Journal of Applied Ecology 37:39-59.

328 Gervasi V, Brøseth H, Gimenez O, Nilsen EB, Linnell JD. 2014. The risks of learning:

329 confounding detection and demographic trend when using count-based indices for population 330 monitoring. Ecology and Evolution 4:4637-4648.

331 Goldberg, CS, Strickler, KM and Fremier, AK. 2018. Degradation and dispersion limit 332 environmental DNA detection of rare amphibians in wetlands: Increasing efficacy of sampling 333 designs. Science of the Total Environment 633:695-703.

334 Gorman TA, Bishop DC, Haas CA. 2009. Factors related to occupancy of breeding wetlands by 335 flatwoods salamander larvae. Wetlands 29:323-329.

336 Guillera-Arroita G, Hauser CE, McCarthy MA. 2014. Optimal surveillance strategy for invasive 337 species management when surveys stop after detection. Ecology and Evolution 4:1751-1760. 
338 Hauser CE, McCarthy MA. 2009. Streamlining 'search and destroy': cost-effective surveillance 339 for invasive species management. Ecology Letters 12:683-692.

340 He F. Gaston KJ. 2000. Estimating species abundance from occurrence. American Naturalist $341 \quad 156: 553-559$.

342 Hedrick PW, Fredrickson R. 2010. Genetic rescue guidelines with examples from Mexican 343 wolves and Florida panthers. Conservation Genetics 11:615-626.

344 Jackson JT, Weckerly FW, Swannack TM, Forstner MRJ. 2006. Inferring absence of Houston 345 toads given imperfect detection probabilities. The Journal of Wildlife Management 70:14613461463.

347 Kellner KF, Swihart RK. 2014. Accounting for imperfect detection in ecology: a quantitative 348 review. PLoS One 9:e111436.

349 Kéry M, Schmidt BR. 2008. Imperfect detection and its consequences for monitoring for 350 conservation. Community Ecology 9:207-216.

351 Korfel CA, Mitsch WJ, Hetherington TE, Mack JJ. 2010. Hydrology, physiochemistry, and 352 amphibians in natural and created vernal pool wetlands. Restoration Ecology 18:843-854.

353 Laikre L, Schwartz MK, Waples RS, Ryman N, GeM Working Group. 2010. Compromising 354 genetic diversity in the wild: unmonitored large-scale release of plants and animals. Trends in 355 Ecology and Evolution 25:520-529.

356 Lebreton JD, Burnham KP, Clobert J, Anderson DR. 1992. Modelling survival and testing 357 biological hypotheses using marked animals: A unified approach with case studies. Ecological 358 Monographs 62:67-118.

359 Manel S, Williams HC, Ormerod SJ. 2001. Evaluating presence-absence models in ecology: the 360 need to account for prevalence. Journal of Applied Ecology 38:921-931. 
361 McConville AJ, Keane A, Milner-Gulland EJ, Grachev IA, Bekenov AB, Coulson T. 2009.

362 Reconstructing the observation process to correct for changing detection probability of a

363 critically endangered species. Endangered Species Research 6:231-237.

364 MacKenzie DI, Nichols JD, Lachman GB, Droege S, Royle AJ, Langtimm CA. 2002. Estimating

365 site occupancy rates when detection probabilities are less than one. Ecology 83:2248-2255.

366 Moore AL, McCarthy MA, Parris KM, Moore JL. 2014. The optimal number of surveys when 367 detectability varies. PloS One 9:e115345.

368 Pacifici K, Dorazio RM, Conroy MJ. 2012. A two-phase sampling design for increasing

369 detections of rare species in occupancy surveys. Methods in Ecology and Evolution 3:721-730.

370 Palis JG. 1997. Breeding migration of Ambystoma cingulatum in Florida. Journal of Herpetology $371 \quad 31: 71-78$.

372 Penteriani V, Otalora F, Sergio F, Ferrer M. 2005. Environmental stochasticity in dispersal areas 373 can explain the 'mysterious' disappearance of breeding populations. Proceedings of the Royal 374 Society B: Biological Sciences 272:1265-1269.

375 R Core Team. 2021. R: A language and environment for statistical computing. R Foundation for 376 Statistical Computing, Vienna, Austria. https:/www.R-project.org/.

377 Regan TJ, McCarthy MA, Baxter PWJ, Dane-Panetta F, Possingham HP. 2006. Optimal 378 eradication: when to stop looking for an invasive plant. Ecology Letters 9:759-766.

379 Rizzo AA, Brown DJ, Welsh SA, Thompson PA. 2017. Factors influencing detection of the 380 federally endangered diamond darter Crystallaria cincotta: implications for long-term 381 monitoring strategies. American Midland Naturalist 178:123-131.

382 Rout TM, Heinze D, McCarthy MA. 2010. Optimal allocation of conservation resources to 383 species that may be extinct. Conservation Biology 24:1111-1118. 
384 Royle JA, Nichols JD. 2003. Estimating abundance from repeated presence-absence data or 385 point counts. Ecology 84:777-790.

386 Royle JA, Nichols JD, Kéry M. 2005. Modelling occurrence and abundance of species when 387 detection is imperfect. Oikos 110:353-359.

388 Scherer RD, Tracey JA. 2011. A power analysis for the use of counts of egg masses to monitor 389 wood frog (Lithobates sylvaticus) populations. Herpetological Conservation and Biology 6:8139090.

391 Semlitsch RD. 1987. Relationship of pond drying to the reproductive success of the salamander 392 Ambystoma talpoideum. Copeia 61-69.

393 Shaffer SA, Roloff GJ, Campa H. 2019. Survey methodology for detecting eastern massasauga 394 rattlesnakes in southern Michigan. Wildlife Society Bulletin 43:508-514.

395 Shea K, Wolf N, Mangel M. 2006. Influence of density dependence on the detection of trends in 396 unobserved life-history stages. Journal of Zoology 269:442-450.

397 Specht HM, Reich HT, Iannarilli F, Edwards MR, Stapleton SP, Weegman MD, Johnson MK, 398 Yohannes BJ, Arnold TW. 2017. Occupancy surveys with conditional replicates: an alternative 399 sampling design for rare species. Methods in Ecology and Evolution 8:1725-1734.

400 Strayer DL. 1999. Statistical power of presence-absence data to detect population declines.

401 Conservation Biology 13:1034-1038.

402 Taylor BE, Scott DE, Gibbons JW. 2006. Catastrophic reproductive failure, terrestrial survival, 403 and persistence of the marbled salamander. Conservation Biology 20:792-801.

404 Veale AJ, Russello MA. 2016. Sockeye salmon repatriation leads to population re-establishment 405 and rapid introgression with native kokanee. Evolutionary Applications 9:1301-1311. 
406 Wintle BA, Walshe TV, Parris KM, McCarthy MA. 2012. Designing occupancy surveys and 407 interpreting non-detection when observations are imperfect. Diversity and Distributions 18:417408424.

409 Zhou S, Griffiths SP. 2007. Estimating abundance from detection-nondetection data for 410 randomly distributed or aggregated elusive populations. Ecography 30:537-549. 
Figure 1

Annual detection probabilities of larval flatwoods salamanders as a function of survey effort.

The solid line indicates the median detection probability for a single year of monitoring, up to 10 surveys per year. Surveys comprise 30 minutes of dipnetting effort, typically shared between multiple surveyors, concentrated in areas of herbaceous vegetation. The dashed lines represent bootstrapped quantiles (0.25-0.75) given annual fluctuations in detectability.

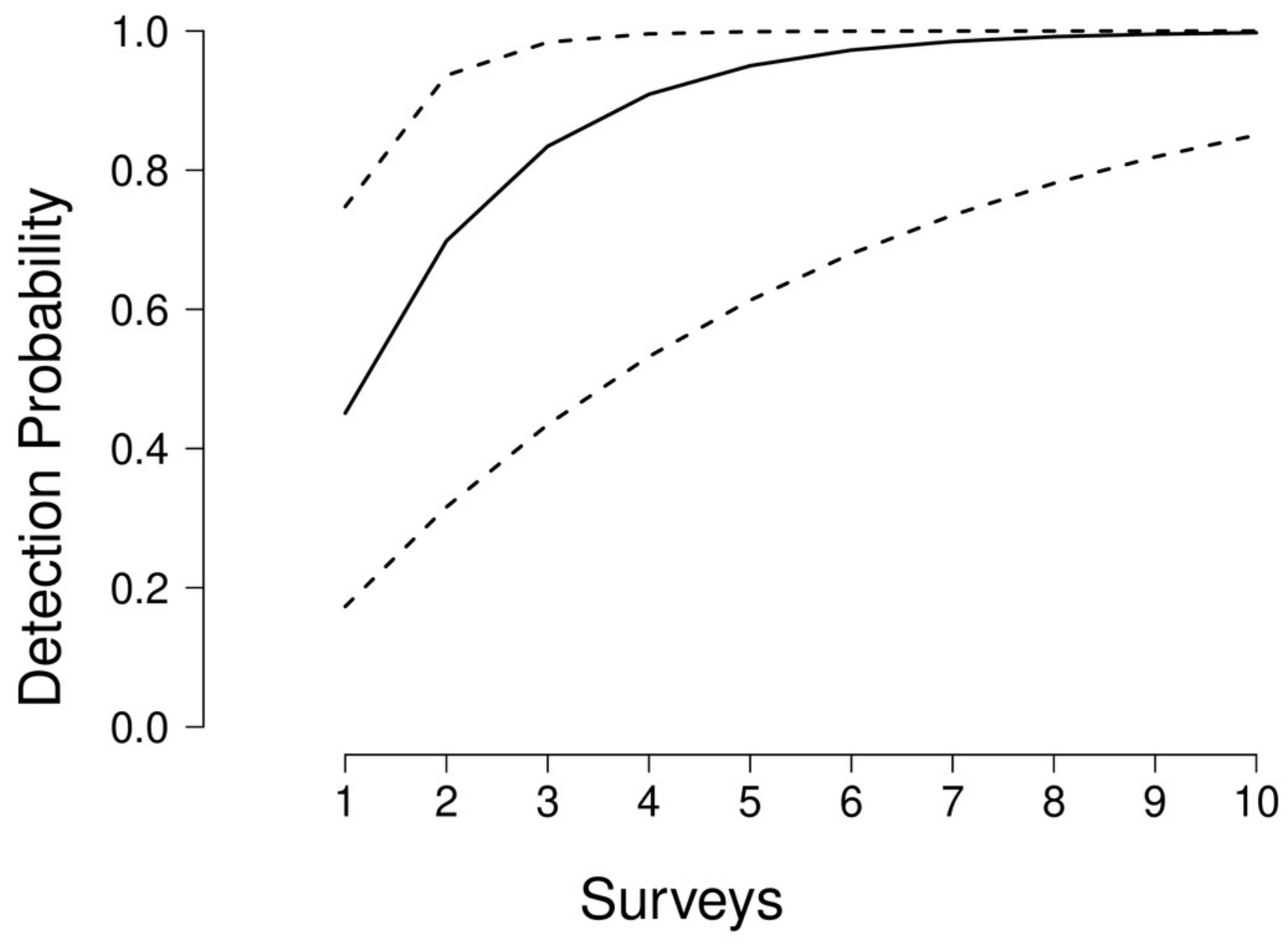




\section{Figure 2}

Cumulative detection probabilities of larval flatwoods salamanders as a function of time and survey effort.

Cumulative detection probabilities are presented for up to ten years of continuous monitoring. The four panels represent different levels of survey effort within each year (1, 2, 3 , or 4 surveys per year). Surveys comprise 30 minutes of dipnetting effort, typically shared between multiple surveyors, concentrated in areas of herbaceous vegetation. The solid lines indicate median detection probabilities and the dashed lines reflect $90 \%$ quantiles (0.05-0.95) given annual fluctuations in detectability. The dot and whisker lines along the bottom of each panel indicate the mean number of years required to achieve a $99 \%$ detection probability and the associated uncertainty. 


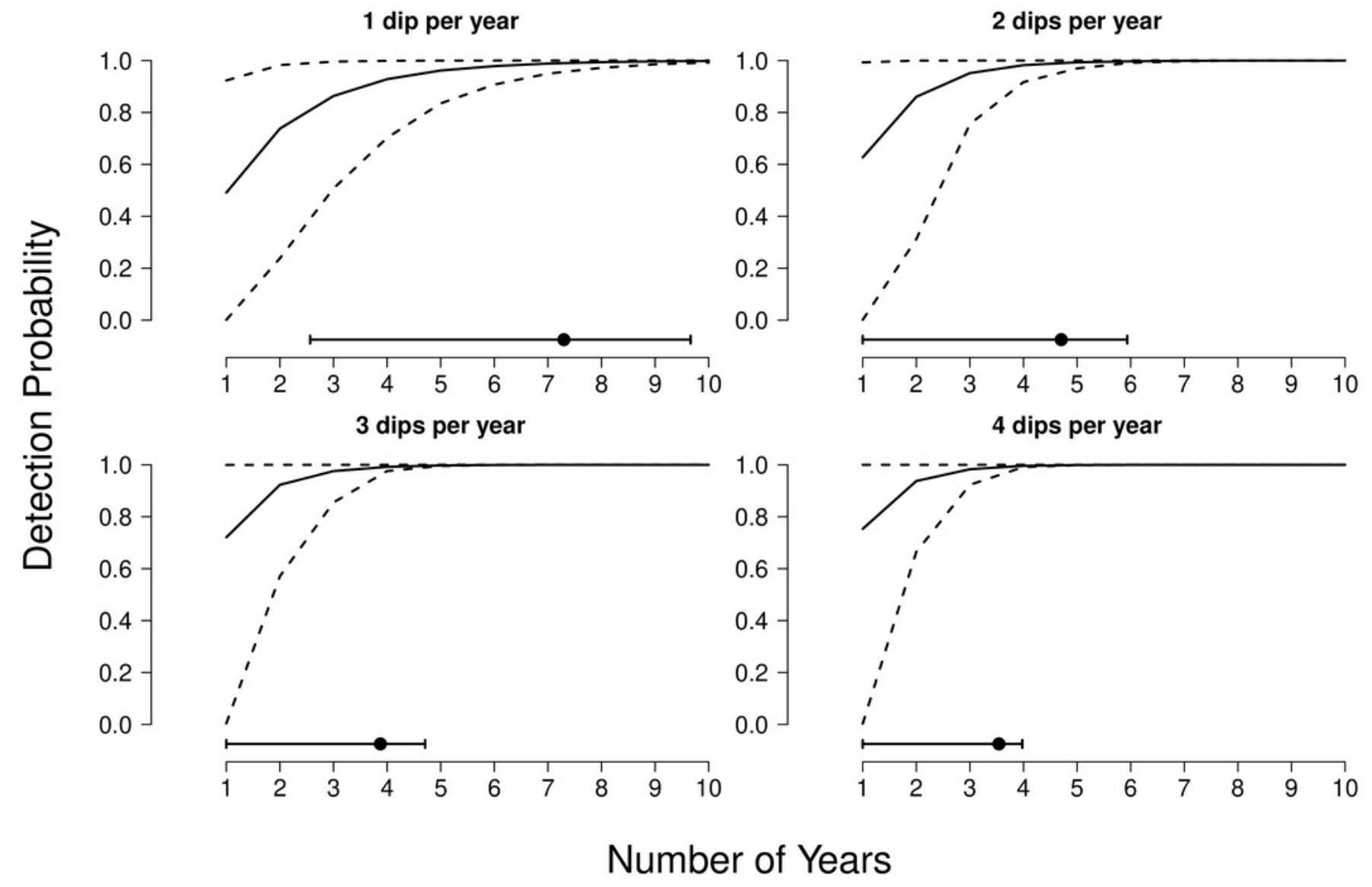

